case of a special system for which $a_{2}, a_{3} \ldots$ are not all $=0$; the investigation of such systems would, I think, be very interesting.

[In my "Memoir on the Rational Transformation between two Spaces," p. 179, line 7 from the bottom, the factor $(3 n-1)$ should be $(3 n-2)$; and p. 180 , eighth line from the bottom, the factor $3 n-4 r+6$ ought to have been $3 n-4 r+4$, viz., the whole term should be diminished by $r(r+1)$. But the "correction," sixth line from the bottom, should be increased by this same quantity, so that the value of the "postulation," as given fourth line from the bottom, is correct.]

A short discussion took place on the above paper; and then Profesisor Cayley gave an account of the following paper:-

\title{
A Second Memoir on Quartic Surfaces.
}

In my Memoir on Quartic Surfaces, ante pp. 19--69, although remarking (See No. 79) that the identification was not completely made out, I tacitly assumed that the symmetroid and the decadianome (each of them a quartic surface with 10 nodes) were in fact identical. There is yet a good deal which I cannot completely explain; but the trnth appears to be, that the decadianome includes two cases of coordinate generality, say the sextic decadianome, and the bicubic decadianome $=$ symmetroid: viz., in the first of these the circumscribed cone, having for vertex any one of the 10 nodes, is a proper sextic cone with 9 double lines; in the second it is a system of two cubic cones, intersecting, of course, in 9 lines, which are double lines of the aggregate sextic cone: or, in the notation of the Table No. 11, in the case of the sextic decadianome, the circumscribed cones are each of them $6_{9}$; in that of the bicubic dianome $=$ symmetroid, they are each of them $(3,3)$. We thus arrive at a very remarkable system of 10 points in space, viz., giving the name "ennead" to any 9 points in plano, which are the intersections of two cubic curves, or to any 9 lines through a point which are the intersections of two cubic cones; the 10 points in space are such that, taking any one whatever of them as vertex, and joining it with the remaining points, the 9 lines form an ennead. I purpose in the present short Memoir to consider the theories in question; the paragraphs are numbered consecutively with those of the Memoir on Quartic Surfaces.

\section{Plune Sertic Curve with 9 Nodes.}

110. A sextic curve contains 27 constants; and the number of con. ditions to be satisfied in order that a given point may be a node is = 3. Hence it would at first sight appear that the curve could be found so as to have 9 given nodes; this would be $9 \times 3,=27$ condi- 
tions, or the curve would be completely determinate. But observe that through the 9 given points we have a determinate cubic curve $\mathrm{U}=0$; we have therefore $\mathrm{U}^{3}=0$ a sextic curve, and the only sextic curve with the 9 given nodes; that is, there is not in a proper sense any sextic curve with the 9 given nodes. The number of given nodes is thus $=8$ at most.

111. The sextic curve with 8 given nodes should contain $27-3.8$ $=3$ constants. We may through the 8 given points draw the two cubics $\mathrm{P}=0, \mathrm{Q}=0$; and we have then $(a, b, c \gamma \mathrm{P}, \mathrm{Q})^{2}=0$, a bicubic, or improper sextic curve having the 8 nodes, and also a ninth node, viz., the remaining point of intersection of the two cubic curves, or say the remaining point of the ennead. Hence if $\nabla=0$ be any particular sextic curve having the 8 given nodes, we have

$$
(a, b, c \gamma \mathrm{P}, \mathrm{Q})^{2}+\theta \nabla=0
$$

a proper sextic curve having the 8 given nodes; and this, as containing the right number $(=3)$ of constants, will be the general sextic curve having the 8 given nodes.

112. There will be a ninth node if $\theta=0$; viz., the curve is then $(a, b, c \gamma \mathrm{P}, \mathrm{Q})^{2}=0$, a bicubic, or improper sextic curve, having for nodes the 9 points of the ennead. Observe that the ninth node is here a point completely and uniquely deterunined by means of the given 8 nodes. Moreover the number of constants is $=2$, so that we have here a general (improper) solution of the question of finding a sextic curve with 9 nodes, 8 of them given.

113. But if $\theta$ is not $=0$, then the ninth node must be a point on the curve $J(P, Q, \nabla)=0$; viz., this is a curve of the order 9 , determined. by means of the given 8 points; say it is the "dianodal curve" of these 8 points, and, as is easy to see, it has each of these 8 points for a node. The ninth node of the sextic may be any point whatever on the dianodal curve; and regarding it as a given point, the sextic will still contain 1 constant; that is, we have the general solution of the problem of finding a sextic curve with 9 nodes, 8 of them given, and the 9 th a given point on the dianodal curve.

114. So long as the 8 points are arbitrary, the dianodal curre does not pass through the 9 th point of the ennead, and the two cases above considered are mutually exclusive. It will be noticed how closely analogous this theory of the plane sextic with 9 nodes, is to that of the quartic surface with 8 nodes.

115. Of course, instead of the plane sextic curve, we may have a sextic cone; such a cone has at most 8 given double lines; and if there be a 9 th double line, then there are the two cases of coordinate generality; viz., (1), the new double line is the ninth line of the ennead, the cone being in this case not a proper sextic cone, but a bicubic cone; (2), the new double line may be any line whatever on the dianodal cone; (cone of the order 9 determined by the 8 given 
lines, and having each of these for a double line,) and regarding it as a given line on the dianodal cone, the sextic cone contains 1 constant.

Each circumscribed cone of the Symmetroid is $(3,8)$.

116. Using $(x, y, z, w)$ as current coordinates of a point of the symmetroid, I take S, T, U, V to be quadric functions of the coordinates $(a, \beta, \gamma, \delta)$; the equation of the symmetroid is therefore given by $x \mathrm{~S}+y \mathrm{~T}+z \mathrm{U}+w \mathrm{~V}=$ cone,

and the nodes thereof are determined by

$$
x \mathrm{~S}+y \mathrm{~T}+z \mathrm{U}+w \mathrm{~V}=\text { plane-pair. }
$$

Suppose that a node is $(x=0, y=0, w=0)$; the condition for this is $V=$ plane-pair ; and we may without loss of generality write $V=\gamma^{2}+\delta^{2}$. Hence, putting for shortness $\theta=x \mathrm{~S}+y \mathrm{~T}+z \mathrm{U}$, that is $\theta$ a quadric function $\quad(a, b, c, d, f, g, h, l, m, n \gamma \alpha a, \beta, \gamma, \delta)^{2}$, wherein the co-efficients $a, b, \ldots$ are arbitrary linear functions of $(x, y, z)$, but not containing $w$, the equation of the symmetroid is given by

$$
\theta+w\left(\gamma^{2}+\delta^{2}\right)=\text { cone. }
$$

117. It follows that the equation is

$$
\left|\begin{array}{l}
a, h, g, l \\
h, b, f, m \\
g, f, c+w, n \\
l, m, n, d+w
\end{array}\right|=0 ;
$$

viz., this is $\quad \nabla+w\left(\delta_{c}+\delta_{d}\right) \nabla+\frac{1}{2} w^{2}\left(\delta^{c}+\delta_{d}\right)^{2}=0$,

where $\nabla$ denotes the foregoing determinant, writing therein $w=0$. Or, observing that $\nabla$ contains $c$ and $d$ each only linearly, the equation may be written $\quad \nabla+w\left(\delta_{c}+\delta_{d}\right) \nabla+w^{2} \delta_{c} \delta_{d} \nabla=0$,

which is a quartic surface having, as it should have, the point $(0,0,0)$ for one of its ten nodes.

118. The equation of the circumscribed cone is

$$
\left(\delta_{c} \nabla+\delta_{d} \nabla\right)^{2}-4 \nabla \cdot \delta_{c} \delta_{a} \nabla=0
$$

or, what is the same thing, it is

$$
\left(\delta_{c} \nabla-\delta_{d} \nabla\right)^{2}+4\left(\delta_{c} \nabla \cdot \delta_{d} \nabla-\nabla \cdot \delta_{e} \delta_{d} \nabla\right)=0 .
$$

But we have identically

so that the equation is

$$
\delta_{c} \nabla \cdot \delta_{d} \nabla-\left(\frac{1}{2} \delta_{n} \nabla\right)^{2}=\nabla \cdot \delta_{c} \delta_{d} \nabla
$$

$$
\left(\delta_{c} \nabla-\delta_{d} \nabla\right)^{2}+\left(\delta_{n} \nabla\right)^{2}=0
$$

a sextic cone breaking up into the two cubic cones

$$
\delta_{c} \nabla-\delta_{d} \nabla \pm i \delta_{n} \nabla=0
$$

so that the cone is $(3,3)$. And since clearly the point $(0,0,0)$ may be regarded as representing any one whatever of the 10 nodes, it follows that for any node whatever of the symmetroid, the circumscribed cone is $(3,3)$, so that, as stated above, bicubio decadianome $=$ symmetroid. 


\section{Deductions from the foregoing theory.}

119. Referring to No. 85 of the original memoir, it appears that, with 6 given points as nodes, we can actually find for the symmetroid an equation containing 6 constants. I cannot discover any ground for doubting that 3 of these may be determined so as to give to the symmetroid a seventh given node; and I therefore assume that with 7 given points as nodes, an equation can be found with 3 constants. The symmetroid is certainly not octadic, hence the eighth node must lie on the dianodal surface of the 7 given points. I can discover no ground for doubting but that two of the constants may be determined so that the eighth node shall be any given point whatever on the dianodal surface of the 7 points; and (this being so) that further the remaining constant may be determined so that the ninth node shall be any given point whatever on the dianodal curve of the 8 points. But if all this be so, the consequence is very remarkable; the tenth node is not any one whatever of the 22 dianodal centres of the 9 points, but it is a uniquely determinate "enneadic centre," viz., we must have the following theorem :-

120. "Take any 7 points ; an eighth point at pleasure on the dianodal surface of the 7 points; a ninth point at pleasure on the dianodal curve of the 8 points. In the system of 9 points so determined, take any one as vertex, and joining it with the remaining 8 , construct the ninth line of the ennead. Performing this construction with each of the 9 points successively as vertex, we obtain 9 lines passing through the 9 points respectively. These 9 lines meet in a point which is the 'enneadic centre' of the 9 points : and further, the 10 points form a completely symmetrical system, so that each one of them is the enneadic centre of the remaining 9."

121. Assuming that the 9 lines do intersect so as to give rise to an enneadic centre, there is no difficulty in conceiving that the loci, which by their intersection determine the dianodal centres, do each of them pass through the enneadic centre; so that this enneadic centre counts once or more among the dianodal centres, and the number of proper dianodal centres, instead of being $=22$, will be suppose $=22-\omega$, and if, further, the 9 points, together with the enneadic centre, are the nodes of a symmetroid, but the 9 points together with any one of the $22-\omega$ dianodal centres are the nodes of a sextic decadianome, then we must also have as follows:

122. "Considering any 9 points as above; taking any one as vertex, and joining it with the remaining 8 , these 8 lines determine a dianodal ninthic cone. We have thus 9 dianodal cones, which cones pass all of them through the same $22-\omega$ points."

123. I am not able to verify these theorems à posteriori. It appears 
to me that the theorem in regard to the enneadic centre subsists for a system of 9 points such as referred to in the statement; but that if by possibility the statement be too general, the theorem must, at all erents, subsist for a more special system of 9 points; and that there certainly exist systems of 10 points, such that each 9 of the points have as an enneadic centre the tenth point. [ $I$ have since ascertained that if a quartic surface with 10 nodes has a single node $(3,3)$, the surface is a symmetroid; whence, by what precedes, the remaining nine nodes are each of them $(3,3)$. Added 25th March.]

124. I notice, as a subject of investigation, the following system of correspondence; viz., given any 8 points in space: then to every point in space corresponds a line through this point: viz., the nintb line of the ennead obtained by joining the point with the 8 given points respectively, and to each line in space a point or points on the line : viz., the point or points for each of which the line is the ninth line of the ennead obtained by joining the point with the eight given points respectively.

Dr. Hirst next entered into an explanation of his paper "On the Polar Correlation of two Planes, and its connection with their Quadric Correspondence."

Profs. Cayley, Smith, Mr. Cotterill, and Dr. Hirst took part in the discussions on the papers.

The following presents were made to the Library :-

"On the determination of the Orbit of a Planet from three Observations." By A. Cayley, F.R.S.: from the author.

"Géométrie Supérieure." By M. Chasles : from the President.

Jan. 12th, 1871.

W. SPOTTISWOODE, Esq., F.R.S., President, in the Chair.

Mr. R. B. Hayward, M.A., late Fellow of St. John's College, and Mathematical Master at Harruw, and the Rev. J. Wolstenholme, M.A., Fellow of Christ's College, were proposed for election.

Mr. Walker gave an account of the following paper:-

On Systems of Tangents to Plane Cubic and Quartic Curves.

1. By a "System of Tangents" I understand all the tangents which can be drawn from a point $\left(x_{1} y_{1} z_{1}\right)$ to a proper plane carre (U). According to Dr. Salmon's notation, the equation to the system of tangents drawn to a cubic curve is obtained by equating with zero the discriminant of the binary cubic in $\lambda, \mu$, (Higher Plane Curves, Ed. 1, p. 68,)

$$
\mathrm{U} \lambda^{3}+\Delta_{1} \lambda^{2} \mu+\Delta \lambda \mu^{3}+U_{4} \mu^{3},
$$

